\title{
Rogue Solutions \& New Open Resources Workgroup Report
}

Bryan Alexander, Lars Bjornshauge, Hillary Corbett, Christopher Erdmann, Nancy Gwinn, Meg Oakley, Paul Peters, Tom Reller, Morgan Stoddard

\section{Abstract / Workgroup Question}

What are the impacts of Sci-Hub and other rogue solutions on open access and what is the future of this approach, which may be gaining new mainstream support (noting for instance Wellcome's recent funding of ResearchGate). What new resources should the scholarly community develop (and how) that would be useful and legal additions to our progress toward open (a new blacklist for instance, or new repositories)? This group will also integrate (to the extent possible) ideas raised by the information overload workgroup from OSI 2016.

\section{Introduction}

The Rogue Solutions \& New Open Solutions workgroup, new to OSI in 2017, began our session by discussing the definition of "rogue" in terms of etymology and practice, teasing out positive and negative connotations. The term suggests opposing established authorities, which really cuts across a wide value spectrum.

The workgroup agreed that Sci-Hub, and any other service that acts in blatant violation of copyright laws, does not fall within the definition of open access and is not a solution to be considered by the workgroup. To get away from the solely negative connotations of "rogue," we decided to coin a more expansive term and asked, what can we learn about scholarly communication from the rise of $\mathrm{New}$ and Entrepreneurial Approaches to Open or...NEATOs? NEATOs are not "rogues" in the legal sense because they operate within the boundaries of the law and are not associated with illegal acts or dishonesty.
Projects discussed included: databases of materials and/or links (LibGen, Sci-Hub); social media venues for sharing articles (r/Scholar on Reddit, \#ICanHazPDF on Twitter); social media in general; web browser extensions (Unpaywall, CanaryHaz, the OA Button). During our meetings, we also touched on Researchgate, CHORUS, repositories (institutional + otherwise), DeepDyve, arXiv + other -Xiv projects, Academia.edu, OpenMinted, Unlatched, and Dissem.in.

\section{Why do NEATO Solutions}

\section{Exist?}

We then explored the question of why rogue of NEATO solutions exist, building on the hypothesis that their proliferation might reveal challenges in the scholarly publication ecosystem. We determined that the reason NEATO projects exist is because readers experience a number of pain points in trying to access published research and scholarship. NEATOs attempt to address these impediments. As

(C) 2017 OSI2017 Rogue Solutions \& New Open Resources Workgroup. This open access article is distributed under the Creative Commons Attribution 4.0 International License. This document reflects the combined input of the authors listed here (in alphabetical order by last name) as well as contributions from other OSI2017 delegates. The findings and recommendations expressed herein do not necessarily reflect the opinions of the individual authors listed here, nor their agencies, trustees, officers, or staff. 
Lars Bjørnshauge of the Directory of Open Access Journals (DOAJ) put it, "We are stuck in a system we want to leave."

Pain points identified by members of the workgroup are:

1. Difficulties in discovering and accessing content

2. Restrictive licensing agreements

3. The diversity of users needing yet unable to obtain access (citizen scientists, global south, etc.)

4. Workflow challenges (navigation, complexity)

5. Digital divide, the gap between the global north and south, the divide between those affiliated with an institution providing access to the full record of scholarly publication versus those who are not, and "the informationally disadvantaged"

6. The sustainable business model challenge

7. Quality vs quantity of materials (following Vint Cerf's opening keynote address)

The workgroup identified several NEATO/rogue solutions (access methods outside of the publisher's traditional distribution channels) that work to improve access and reduce costs. It's important to recognize that a number of NEATOs, including Unpaywall, OA Button, Dissem.in, arXiv.org, Sci-Hub, et al., occupy different points across a spectrum of legality.

\section{How Can NEATO Solutions}

\section{Help?}

The workgroup then explored and discussed how these projects could contribute to a solution space. At this point we only considered NEATO solutions that do not violate copyright laws. This led us to several possible models:

Pay-per-view reform: $a / k / a$ "iTunes for research papers." This is a recognizable business model, and one that guarantees payment and quality control. However, it may not actually be open in many senses of the term. Publishers might not see an economic incentive to migrate to such a system, given their current business model's success; indeed, publishers might lose some control.

Subscription access reform: $a / k / a$ "Netflix for scholarly publication." There are some examples of this already in play, such as DeepDyve. However, there are cost issues, as the price point can still exclude audiences. Low adoption levels can also render projects inviable.

Open access gateway services: Projects like $\underline{1 \text { Science }}$ and ScienceOpen organize OA content in one spot. They are also legally sound. However, their value proposition isn't necessarily clear to purchasers, and adoption rates remain low.

Niche projects based on open: There are a number of projects grounded in narrow scholarly communities that use open access to serve them well, such as the $\underline{\mathrm{Bi}}-$ odiversity Heritage Library (Smithsonian Museums) and the SAO/NASA Astrophysics Data System (Harvard University and the Smithsonian).

Artificial intelligence: The workgroup also looked at new, emerging or potential NEATO examples that employ artificial intelligence, such as Meta, recently acquired by the Chan Zuckerberg Initiative. The potential advantages of solutions in- 
corporating AI are greater efficiency and accuracy in addressing quality issues and enhancing the user experience.

Meta... hopes, by bending artificial intelligence to the task, to identify important papers from the $2 \mathrm{~m}$ or so produced every year. The firm's computers have attempted to recognise features of widely cited papers that contributed to their success. Sam Molyneux, Meta's boss, claims that as a result the firm's software can now predict the impact of newly published work. ${ }^{1}$

To aid the accuracy of the algorithms, increasing available OA content will be critical. Using AI to assess research, however, also raises potential concerns for authors and others, including:

1. Is it possible or desirable for a black box algorithm to replace scholarly expertise?

2. Faculty are likely to be resistant to AI due to fears of possible ulterior motives or data manipulation (predictive analytics warping output, downplay low-scoring materials unfairly, and suspicion of the Zuckerberg's-Facebook connection)

Additionally, we considered the possibility of someone creating an automated scholarly content quality assessment tool, in order to at least help libraries and readers avoid fake journals.

Global OA flip: Another entrant in the solution space was the possibility of a global flip to a gold OA paradigm, particularly in light of the recent of Harvard Library report studying this option in depth. Like our other NEATO solutions, a global flip raises several questions:

1. Will those scholars who cannot pay APCs be disadvantaged in getting their work published? (i.e., access remains an issue)

2. What will be the incentive for researchers to change?

3. Will the scholarly workflow become more complex?

4. Will this present a challenge to librarians' roles in selecting and making available materials?

Journal Master List: The final entrant the workgroup considered for the solution space was the creation of a Journal Master List, which would improve knowledge of what users and institutions already have access to and could be a short-term fix in a transition towards Gold OA. This NEATO solution, possibly in combination with automated assessment tools, could address information overload and quality issues. The Journal Master List could include both a journal whitelist and a journal blacklist.

\section{Conclusions}

The bottom line is that there is no "killer app" that will magically solve the problems outlined in Part II. NEATOs/rogues are indeed instructive in identifying pain points and possible, if limited solutions. However, when it comes to addressing large scale problems in scholarly publication or advancing the cause of open, these NEATOs point to the necessity of massive, cultural transformation, rather than the promulgation of marginal projects. We acknowledge that some NEATOs, like SciHub, are too dangerous/illicit to be 
supported. Others have great potential but require individual action (installation of browser extensions) or are limited to what's already freely available to read. The wild card to follow in this space is AI.

\section{Additional Resources}

Presentation slides: $\underline{\text { http:/ / bit.ly/osi2017neato }}$

Storify of Tweets: https://storify.com/bryanalexander/rogue-solutions-for-scholarlypublication

\section{Rogue Solutions \& New Open Resources Workgroup}

Bryan Alexander, President, Bryan Alexander Consulting

Lars Bjørnshauge, Founder and Managing Director, DOAJ

Hillary Corbett, Director of Scholarly Communication \& Digital Publishing, Northeastern

University

Christopher Erdmann, Chief Strategist for Research Collaboration, NCSU Libraries

Nancy Gwinn, Director, Smithsonian Institution Libraries

Meg Oakley, Director of Copyright \& Scholarly Communications, Georgetown University

Paul Peters, CEO, Hindawi

Tom Reller, Vice President Global Corporate Relations, Elsevier

Morgan Stoddard, Director of Research Services, George Washington University

\section{End Notes}

\footnotetext{
1 "Assessing the importance of scientific work," The Economist, March 23, 2017 (As of August 2, 2017: http://www.economist.com/news/science-and-technology/21719441alternative-metrics-extend-concept-citation-beyond-journal
} 